\title{
A review of non-strabismic accommodative-vergence anomalies in school-age children. Part 1: Vergence anomalies
}

\author{
Authors: \\ Samuel O. Wajuihian ${ }^{1}$ \\ Rekha Hansraj ${ }^{1}$ \\ Affiliations: \\ ${ }^{1}$ Department of Optometry, \\ University of KwaZulu-Natal, \\ South Africa \\ Correspondence to: \\ Samuel Wajuihian \\ Email: \\ swajuihian@mweb.co.za \\ Postal address: \\ Private Bag X54001, Durban \\ 4000, South Africa \\ Dates: \\ Received: 24 Feb. 2014 \\ Accepted: 17 Dec. 2014 \\ Published: 28 Aug. 2015 \\ How to cite this article: \\ Wajuihian SO, Hansraj R. \\ A review of non-strabismic \\ accommodative-vergence \\ anomalies in school-age \\ children. Part 1: Vergence \\ anomalies. Afr Vision Eye \\ Health. 2015;74(1), Art. \\ \#32, 10 pages. http://dx.doi \\ org/10.4102/aveh.v74i1.32

\section{Copyright:} \\ (C) 2015. The Author(s). \\ Licensee: AOSIS \\ OpenJournals. This work is \\ licensed under the Creative \\ Commons Attribution \\ License.
}

\section{Read online:}

Comfortable reading and the performance of near point activities involve efficient accommodative and vergence systems. However, accommodative and vergence anomalies are associated with various symptoms that impair efficient near point tasks. Although several studies investigated accommodative-vergence anomalies in school-age populations, their findings were diverse owing to differences in diagnostic techniques and the criteria used to define the variables. The aim of this paper is to derive prevalence and distribution estimates of vergence anomalies in school-age children and address variations in the study methods and findings. Despite variations in the study methods and findings, accommodativevergence anomalies were common in school-age populations. Variations and limitations of previous studies are discussed and recommendations for improving future studies are suggested.

\section{Introduction}

Non-strabismic accommodative and vergence binocular anomalies affect clarity and binocularity, and impair comfort and efficiency of visual performance when near tasks such as reading, writing and computer-based work is performed. . $^{1,2,3,4,5}$ Learning involves reading, which is the process of extracting meaning from written text and is a fundamental part of a child's education. ${ }^{6}$ A significant proportion of a child's activities in the classroom ${ }^{7,8}$ and at home ${ }^{8}$ involve the accommodative and vergence mechanism. Accommodative-vergence anomalies (AVAs) result from imbalances between these functions, and the anomalies are aggravated by prolonged visually demanding near tasks, resulting in symptoms. ${ }^{2,9,10,11,12,13}$ These symptoms (Table 1) 10,11,12,13 cause discomfort, impair efficient near tasks and affect overall quality of life., ${ }^{9,10,11,12,13}$ For school-age children and especially high school learners, such symptoms tend to increase as pupils progress through school and demand increases of their accommodative-vergence system for sustained clear vision, as a result of prolonged reading and increased information processing. ${ }^{1,2,3,4,5}$

AVAs have several negative consequences that further highlight their clinical importance. Several studies $^{9,10,14}$ have reported on the association of AVAs with some behavioural and learning problems. Granet et al. ${ }^{9}$ found that convergence insufficiency $(\mathrm{CI})$ is three times more common in patients with attention deficit hyperactivity disorder (ADHD) and that five out of the nine clinical criteria used to diagnose ADHD are identical in CI. Borsting et al. ${ }^{10}$ also found that children with accommodative and non-strabismic binocular anomalies have a higher frequency of ADHDlike behaviours. Similarly, Grönlund et al. ${ }^{15}$ demonstrated an association between near vergence disorders and ADHD. A review by Damari et al. ${ }^{16}$ found that binocular vision anomalies are often misdiagnosed as ADHD, whilst some studies reported that children who have binocular anomalies experience anxiety, emotional and social problems. ${ }^{14,17}$ Furthermore, children with uncorrected AVAs may be misdiagnosed as being dyslexic, ${ }^{18,19,20,21,22}$ and AVAs are sometimes associated with lowered academic achievements in school-age children. ${ }^{19}$

Vision screening has been a traditional way of identifying vision anomalies in school children. However, such screenings may be inadequate, and are limited to detecting reduced visual acuity and refractive errors without testing for accommodative and vergence anomalies. ${ }^{1,2}$ Prescription spectacles recommended for refractive compensation may be inadequate for AVAs. ${ }^{1,2}$ Overall, epidemiological studies of AVAs are significant in enabling identification of individuals who require intervention, and also guiding clinicians and researchers in understanding the prevalence, and characteristics of AVAs. Clues towards determining the possible aetiology of such anomalies by identifying the associated risk factors may become clearer. ${ }^{23,24,25}$ In addition, epidemiological studies are useful in health policy planning, resource allocation, advocacy for increased awareness of the anomalies, and generating hypotheses for future studies..$^{23,24,25}$ Studies 
TABLE 1: Symptoms in vergence anomalies.

\section{Symptoms}

Blurred vision for near tasks or worse after near work

Headaches/eyestrain/dull orbital pain/pulling sensation around the eyes Loss of comprehension/avoidance of near work

Watering or conjunctival or eyelid irritation, sensitivity to light

Eye fatigue/dizziness/sleepiness

Blurred vision worse after reading/near work

Difficulty focusing from far to near or near to far

Hold reading material close or farther way

Difficulty sustaining attention on near point tasks

Letters or words appearing to float or move around

Postural changes noted when working at a desk, or frowns during near tasks

Spatial distortion in size, shape or inclination of objects

on AVAs may identify anomalies that may not present with symptoms, especially in children who may be poor at characterising symptoms. ${ }^{26,27,28}$ Accommodative-vergence anomalies are often detected when a binocular evaluation is performed. Accordingly, given the clinical importance of AVAs, the main aim of this review is to document the prevalence and distribution of AVAs in school-age children. A secondary aim is to highlight the variations and possible limitations of previous studies, which will guide subsequent studies.

\section{Methods and scope of review}

The electronic databases searched included PubMed, Medline, Science Direct, Google Scholar, EBSCO, and Embase using the terms convergence insufficiency, convergence excess, fusional vergence dysfunction, vergence anomalies, heterophoria and schoolchildren. Relevant articles on reference lists were identified and retrieved from electronic and print journals. A manual search of tables of contents of print and electronic versions of optometry journals at the University of KwaZuluNatal library was also conducted and, when necessary, interlibrary loan facilities were utilised. Over $90 \%$ of the reviewed articles were obtained by manual identification on reference lists in published papers. An article was considered for review if it reported on the epidemiology (prevalence and distribution) of vergence anomalies, was a primary research article published in a peer-reviewed journal, and was reported in the English language.

For the present review, studies were first profiled individually, followed by comparisons and synthesis with other studies whilst the classification criteria, measurement techniques and sources of variations and limitations of studies are discussed. The review is of school-age children between 6 and 18 years of age, and the focus is on aspects of the epidemiology of AVAs. A few classic papers published in the early $1990 \mathrm{~s}^{29,30,31,32,33,34}$ were considered, so as to relate previous concepts to current understanding. The review is presented in two parts. In Part 1, a review of studies on vergence anomalies such as $\mathrm{CI}$, convergence excess (CE), fusional vergence dysfunction (FVD) and heterophoria (phoria) are reviewed. In Part 2, accommodative anomalies such as accommodative insufficiency (AI), accommodative excess (AE) and accommodative infacility (AIF) are reviewed.

\section{Vergence}

Vergence is the simultaneous rotational movement of the eyes in opposite directions to obtain or maintain single binocular vision. ${ }^{13,35}$ In vergence anomalies, the eyes are unable to fix and to stabilise a retinal image accurately. The visual axes may move toward each other (convergence) or away from each other ${ }^{13,35}$ (divergence). Vergence anomalies of interest to the current review include CI, CEs, FVD and heterophoria.

Scheiman et al. $^{25}$ reported that, beside refractive errors, the most common vision anomalies in children below 18 years of age are accommodative-convergence anomalies. Although the assessment of an individual's AVA status constitutes an important part of optometric practice and research, studies in this area of eye care show diverse findings owing to the methodological challenges in conducting such studies on a large scale. ${ }^{24,25}$ However, several studies $18,25,26,28,37,38,39,40,41,42,43,44,45,46,47,48,49,50,51,52,53,54,55,56,57,58,59$ on various racial groups have studied vergence anomalies in school-age children.

\section{Convergence insufficiency}

Convergence insufficiency is a binocular anomaly characterised by an inability to attain or maintain adequate convergence without excessive effort. ${ }^{12,13,36}$ Being the most common vergence anomaly in school-age populations, ${ }^{12}$ $\mathrm{CI}$ is of great clinical importance and a syndrome that is diagnosed on the basis of several clinical signs and symptoms. 12,13,27,29,30 The diagnostic techniques applied by studies on vergence anomalies varied, although those commonly used were cover test (CT), von Graefe (VG) method for heterophoria measurement, and the push-up-to-break technique for measurement of near point of convergence (NPC). Fusional vergences were assessed using the step vergences (prism bar) or the smooth vergences (phoropter). Diagnostic signs of CI include exophoria greater at near than at far, reduced positive fusional vergence ranges $(\mathrm{PFC})$ at near, receded NPC, ${ }^{12,29,30,37,38,39}$ low accommodative-convergence (AC/A) ratio, low negative relative accommodation (RA) and high findings on MEM. ${ }^{12,37,38,39}$ The Convergence Insufficiency Reading Study (CIRS) group $37,38,39$ is a research group whose aim is to standardise the conduct of research in CI. The clinical signs and diagnostic system for the diagnosis of CI recommended by the CIRS group ${ }^{37,38,39}$ include:

- exophoria at near

- exophoria at near that is $\geq 4$ pd greater in magnitude than the distance phoria

- insufficient positive fusional vergence (PFV): fails Sheard's criteria or poor PFV at near $\leq 12$ pd base-out (BO) blur or

- $\leq 15$ pd BO break

- receded NPC $\geq 7.5 \mathrm{~cm}$ break or $\geq 10.5 \mathrm{~cm}$ recovery.

To determine if a patient is symptomatic, a score of 16 is required using the convergence insufficiency symptoms 
survey (CISS). ${ }^{40}$ In addition to the clinical criteria, the CIRS recommended a classification system such as:

- low suspect (exophoria at near greater than at far of greater than or equal to 4 prism diopters and clinical sign \#1 above)

- high suspect (exophoria at near and 2 signs, or clinical sign \#1 and \#2 plus \#3 or \#4 above)

- definite (exophoria at near and 3 signs, or all clinical signs \#1-4 above).

The CIRS group classification does not prioritise the traditional definition of receded NPC.

\section{Previous studies on convergence insufficiency}

The findings from reviewed studies are diverse mainly because of variations in the criteria applied to define anomalies; one approach that was applied to facilitate comparison of the findings was to present the review based on the type of population from which the samples were derived, such as non-clinical (unselected) $37,38,41,43,44,46,47,48,49,50,51,52,53,54,55,56,57,58,59$ or clinical (selected) 1, $25,26,38,42$ Another approach was to classify studies on the basis of the number of clinical signs applied to define VAs (single or several clinical signs). With single criteria, CI was defined using only one clinical sign such as receded NPC or exophoria at near. With several signs such as the CIRS classification criteria, the single criterion was applied in addition to other clinical signs.

\section{Studies conducted on non-clinical settings}

The reviewed studies on non-clinical populations ${ }^{37,38,41,43,44,46}$ were conducted mainly in school settings. Such studies enable random sampling, improve generalisations of findings, and offer the merit of testing children in environments similar to those in which they learn. Seven studies $43,49,51,53,54,56,57$ on unselected populations that applied the single criteria of receded NPC and using the push-up-to-break technique are discussed. Letourneau et al. ${ }^{56}$ investigated the relation between CI and school achievement in a sample of 735 schoolchildren aged between 7 and 14 years. Using the criteria of NPC $\geq 10 \mathrm{~cm}$ to make a diagnosis, the prevalence of $\mathrm{CI}$ found was $8.3 \%$ and there was no correlation between CI and any measure of school achievement assessed. For Swedish children aged between 6 and 16 years, Abdi and Rydberg ${ }^{53}$ found that $18 \%$ of the participants in their study had NPC $\geq 10 \mathrm{~cm}$. Using a study method similar to Abdi and Rydberg ${ }^{53}$, Abdi et al. ${ }^{54}$ reported a $6 \%$ (defined as NPC $\geq 10 \mathrm{~cm}$ ) prevalence of CI in a study of participants aged 6-16 years, selected from 8 elementary schools in Sweden. Similar to the findings by Abdi et al. ${ }^{54}$ Harris $^{43}$ conducted a vision screening of 171 elementary school children in Baltimore City and found that only $6 \%$ of the study participants had CI defined as NPC between $8 \mathrm{~cm}$ and $10 \mathrm{~cm}$. The percentage of children with poor convergence increased with age.
Three studies ${ }^{49,50,51}$ in Australia adopted the single criterion for defining CI. In a study of 877 Australian schoolchildren between the ages of 6 and 11 years, Macfarlene et al. ${ }^{50}$ reported a $6.5 \%$ prevalence of CI defined as NPC $\geq 6 \mathrm{~cm}$. Another study in Australia comprised a large-scale vision screening of 2697 children aged between 3 and 12 years by Junghans et al. ${ }^{51}$ who found that $11.2 \%$ of children had NPC $\geq$ $7.5 \mathrm{~cm}$ whilst $11 \%$ had NPC $\geq 10 \mathrm{~cm}$. In a third study in Australia, Walters ${ }^{49}$ conducted a screening of 5597 Australian schoolchildren, and reported a relatively high prevalence (18.1\%) for NPC $\geq 10 \mathrm{~cm}$ which was similar to the prevalence of $18.1 \%$ found by Abdi and Rydberg ${ }^{53}$ in Sweden. Taken together, studies that defined CI using the NPC single criteria may limit interpretation because current literature describes $\mathrm{CI}$ as a syndrome of clinical signs, and single diagnostic criteria may therefore be inadequate to warrant a definite diagnosis of CI. However, a single criterion may be relevant in symptomatic patients whose evaluations indicate that other binocular vision parameters are normal.

Some studies ${ }^{37,39,41,44,51,57}$ conducted on unselected populations of schoolchildren adopted the multiple clinical signs. Rouse et al. ${ }^{37}$ conducted a pilot study to evaluate $\mathrm{CI}$ in 35 fifth- and sixth-graders selected from a private school in the USA. The prevalence estimates reported were: low suspect, $n=8(33.3 \%)$; high suspect, $n=1(4.2 \%)$ and definite, $n=1(4.2 \%)$. The prevalence of clinically significant CI reported by Rouse et al. ${ }^{37}$ was $8.4 \%$. Another study by Rouse et al. ${ }^{39}$ comprised 453 fifth- and sixth-grade schoolchildren aged between 9 and 13 years. Participants were recruited from 3 schools in 3 different locations (California, Chicago and Philadelphia). Heterophoria was evaluated using the von Graefe technique, NPC was measured using the push-up technique with accommodative target, and fusional vergences were assessed using the von Graefe technique. Rouse et al. ${ }^{39}$ reported the total prevalence of CI to be $21.4 \%$, classified as: low suspect $(8.4 \%)$, high suspect $(8.8 \%)$ and definite $(4.2 \%)$. Borsting et al. $^{41}$ investigated the association of symptoms and $\mathrm{CI}$ and AI in 392 children aged 8-15 years. Participants were recruited from 4 schools in the USA. Heterophoria was assessed using the cover test, NPC was measured using the push-up-to-break technique with an accommodative target, and fusional vergences were evaluated using the prism bar in free space at $30 \mathrm{~cm}$. The criteria for PFV were Sheard's criterion, and less than or equal to 7 prism diopters (pd) for break or $3 \mathrm{pd}$ for recovery. These criteria are different from the CIRS criteria (PFV at near less than or equal to $12 \mathrm{pd} \mathrm{BO}$ blur or less than or equal to $15 \mathrm{pd}$ base-out break). The criteria for fusional vergences were derived from the participants from their study who had normal binocular vision values. The total prevalence of CI was $17.3 \%$, of whom about $4.6 \%$ had three-sign (definite) CI, whereas $12.7 \%$ had 2 signs (high suspect) CI, and there was no report on low suspect CI. A third study that used the CIRS multiple criteria was conducted by Marran et al., ${ }^{44}$ and was similar to that of Borsting et al. ${ }^{41}$ Marran et al. ${ }^{44}$ studied convergence CI and AI in relation to symptoms in 299 schoolchildren (mean age 11.5 years, s.d. \pm 0.63 ). The study participants were randomly selected from 19 elementary schools in the 
USA. NPC was assessed using the push-up-to-break technique with an accommodative target; heterophoria was measured using the von Graefe technique; and fusional vergences were evaluated using prism bars. The prevalence of CI was found to be $14.7 \%$ for CI types 2 and 3 .

A second study in Canada defined CI using 2 clinical signs (exophoria greater at near than distance, and NPC $>10 \mathrm{~cm}$ ). In that study, Letourneau and Ducic ${ }^{57}$ investigated the prevalence of $\mathrm{CI}$ in 2084 schoolchildren aged between 5 and 13 years. The participants for the study were randomly selected from 6 elementary schools in Montreal. NPC was measured using the push-up-to-break technique with a penlight target, and heterophoria was assessed using the cover test. The prevalence of CI was $2.2 \%$. Although the magnitude of heterophoria used in the classification was not specified, the criteria applied were similar to the CIRS classification as low suspect CI, which is exophoria at near greater than distance and an additional clinical sign. The higher prevalence $(8.3 \%)$ in the earlier study by Letourneau et al. ${ }^{56}$ compared with the lower prevalence of $2.2 \%$ found by Letourneau and Ducic ${ }^{57}$ might be because of the single criterion of receded NPC used to classify CI in the earlier study, whereas Letourneau and Ducic ${ }^{57}$ used 2 criteria. The lack of information on the magnitude of heterophoria in Letourneau and Ducic ${ }^{57}$ limits the interpretation.

Another study that applied the CIRS group's classification system was reported by Junghans et al. ${ }^{51}$ Heterophoria was measured at a near distance of $33 \mathrm{~cm}$ using a Howell phoria card. It was reported that only $2.1 \%$ of their sample of Australian schoolchildren aged between 3 and 12 years had definite $\mathrm{CI}$, whereas $5.9 \%$ had low suspect $\mathrm{CI}$, which is lower than the prevalence of $8.4 \%$ for low suspect CI reported by Rouse et al. ${ }^{39}$ and the $33.3 \%$ reported by Rouse et al. ${ }^{37}$ Junghans et al..$^{51}$ suggested that the differences in their report compared with the study by Rouse et al..$^{39}$ related to the ethnic diversity of the populations, as there are few black and Hispanic people in Australia, but many Asians. The difference in age in the samples studied is another possible source of variation in the findings between these studies. ${ }^{39,51,41}$ In Junghans et al., ${ }^{51}$ the classification of low, high and definite CI appears to be inconsistent, as a poor fusional reserve which is a clinical sign required to classify $\mathrm{CI}^{38,39}$ was not considered in that study. ${ }^{51}$

Shin et al..$^{55}$ studied accommodative and vergence anomalies in 114 South Korean schoolchildren of between 9 and 13 years old. Participants for the study were recruited from a public school. NPC was measured with the pushup-to-break technique using the fixation stick and a millimetre ruler. Heterophoria and fusional reserves were measured using the von Graefe technique. Multiple signs were applied to define $\mathrm{CI}$ although a $6 \mathrm{pd}$ difference between near and distance exophoria was applied instead of a $4 \mathrm{pd}$ difference in the CIRS criteria. The prevalence of CI was $28 \% .{ }^{55}$ Furthermore, Shin et al. ${ }^{55}$ noted that binocular dysfunctions were significantly associated with impaired academic performance. Interestingly, Shin et al..$^{55}$ used relatively stringent criteria yet found a high prevalence of CI. However, data collection from a single school, as in the study by Shin et al., ${ }^{55}$ limits generalisation to a larger population.

Two studies ${ }^{28,59}$ were reported on South African populations. Metsing and Ferreira ${ }^{59}$ assessed vergence functions in 78 3rdand 4th-grade schoolchildren in Johannesburg, South Africa. There was no report on convergence insufficiency, excess and fusional vergence dysfunction as those were not part of the aims of the study. However, the authors found that $17 \%$ of the participants had poor convergence amplitude, and $21.9 \%$ had poor vergence facility. In a pilot study comprising 65 high school learners, Wajuihian and Hansraj ${ }^{28}$ reported that $16 \%$ had low suspect CI, no participant had high suspect CI and $1.6 \%$ had definite CI. The possible limitations of both studies are the small sample size whilst sample selection using convenience sampling by Metsing and Ferreira might limit generalisation.

Taken together, for the studies that applied the multiple signs on unselected populations of schoolchildren, Borsting et al. ${ }^{41}$ and Rouse et al. ${ }^{39}$ reported a similar prevalence of $4.6 \%$ and $4.2 \%$ respectively for three-sign CI. For the total of CI types 2 and 3 (clinically significant CI), Marran et al. ${ }^{44}$ and Borsting et al. $^{41}$ reported a higher prevalence of $14.7 \%$ and $17.3 \%$ respectively than Rouse et al. ${ }^{39}$ who reported a $13 \%$ prevalence. However, Borsting et $\mathrm{al}^{41}$ found a higher prevalence $(12.7 \%)$ for two-signs CI than Rouse et al..$^{39}(8.8 \%)$. The difference in the findings between the three studies is related to the method and criteria used to measure and define positive fusional vergence. Borsting et al. ${ }^{41}$ and Marran et al. ${ }^{44}$ used prism bars whereas Rouse et al. ${ }^{39}$ used the von Graefe technique. More so, Borsting et al. ${ }^{41}$ used a test distance of 30 $\mathrm{cm}$ instead of $40 \mathrm{~cm}$. The change in test distance is significant, as moving the testing distance from $40 \mathrm{~cm}$ to $30 \mathrm{~cm}$ causes an approximately $5 \mathrm{pd}$ reduction in the positive fusional range. Furthermore, fusional vergence measurement varies with the technique used, and step vergence testing using the prism bar has been reported to give a higher base-out break value at near than the von Graefe technique. ${ }^{60,61}$ It is unclear why the $33 \%$ prevalence for low suspect CI found in the earlier study by Rouse et al. ${ }^{37}$ was much higher than the values reported by Rouse et al., ${ }^{39}$ for which the same study design was used although the study in 1995 was a pilot study.

\section{Studies conducted on non-clinical populations}

In some studies, data were collected from clinical settings either prospectively ${ }^{25}$ or retrospectively ${ }^{38,42}$ from patients who attended university optometry clinics ${ }^{25,38,42}$ or from independent optometric practices. ${ }^{18,26}$ Prospective subject recruitment and data collection has merit over retrospective analysis because the study can be designed to reduce bias. From the study on prospective clinical data, Scheiman et $a .^{25}$ studied the prevalence of vision and ocular disease conditions in a clinical paediatric population. The study participants were consecutive patients who attended the Pennsylvania 
TABLE 2: Summary of studies on convergence insufficiency.

\begin{tabular}{|c|c|c|c|c|}
\hline Authors/year of study & Country of study & Age (years) & Sample size & Prevalence (\%) \\
\hline Letourneau et al. $(1979)^{56}$ & Canada & $7-14$ & 735 & 8.3 \\
\hline Abdi and Rydberg $(2006)^{53}$ & Sweden & $6-16$ & 120 & 18 \\
\hline Abdi et al. $(2008)^{54}$ & Sweden & $6-16$ & 216 & 6 \\
\hline Macfarlane $(1987)^{50}$ & Australia & $6-11$ & 877 & 6.5 \\
\hline Junghans et al. $(2002)^{51}$ & Australia & $3-12$ & 2697 & 11 \\
\hline Walters $(1982)^{49}$ & Australia & $\mathrm{n} / \mathrm{a}$ & 5597 & 18.18 \\
\hline Rouse et al. $(1995)^{37}$ & USA & $9-13$ & 35 & Low suspect $\mathrm{Cl}=33.3$; high suspect $=4.2 ;$ definite $\mathrm{Cl}=4.2$ \\
\hline Rouse et al. (1999) ${ }^{39}$ & USA & $9-13$ & 453 & Low suspect $\mathrm{Cl}=8.4$; high suspect $\mathrm{Cl}=8.8$; definite $\mathrm{Cl}=4.2$ \\
\hline Borsting et al. $(2003)^{41}$ & USA & $8-15$ & 392 & High suspect + definite $\mathrm{Cl}=17.3 \%$ \\
\hline Marran et al. $(2006)^{44}$ & USA & 11.5 & 299 & High suspect $\mathrm{Cl}+$ definite $=4.7$ \\
\hline Junghans et al. $(2002)^{51}$ & Australia & $3-12$ & 2697 & Low suspect $\mathrm{Cl}=5.9$; definite $\mathrm{Cl}=2.1$ \\
\hline Wajuihian and Hansraj (2014) ${ }^{28}$ & South Africa & $13-19$ & 65 & Low suspect $\mathrm{Cl}=16$; definite $\mathrm{Cl}=1.6$ \\
\hline Rouse et al. $(1998)^{38}$ & USA & $8-12$ & 415 & Low suspect $\mathrm{Cl}=33$; high suspect $\mathrm{Cl}=12$; definite $\mathrm{Cl}=6$ \\
\hline Shin et al. $(2009)^{55}$ & South Korea & $9-13$ & 114 & 28 \\
\hline Scheiman et al. $(1996)^{25}$ & USA & $6-18$ & 1650 & 5.3 \\
\hline Dwyer $(1992)^{26}$ & $7-18$ & 144 & 33 & - \\
\hline Dusek et al. 2010) ${ }^{18}$ & $6-14$ & 328 & 5.2 & - \\
\hline Letourneau and Ducic (1988) ${ }^{57}$ & $5-13$ & 2084 & 2.2 & - \\
\hline
\end{tabular}

College of Optometry Clinic. A total of 2023 children between 6 months and 18 years of age (1650 children were between 6 years and 18 years) were enrolled in the study. NPC was measured using the push-up-to-break technique and a penlight target; heterophoria was measured with the cover test; and the technique for fusional reserves was not indicated. Four of the 11 diagnostic signs indicated were used to classify CI. The prevalence of CI in the school-age group was $5.3 \%$, which is higher than the $1.6 \%$ prevalence found in the preschool children in the same study by Scheiman et al..$^{25}$ The difference in prevalence between both age groups may be related to fewer near task demands in younger children, as $\mathrm{CI}$ has been noted to increase with an increase in near task demands. ${ }^{62}$

A second study on a clinic population was conducted by Rouse et al. ${ }^{38}$ The authors studied the frequency, distribution and characteristics of CI in a sample of 8- to 12-year-old schoolchildren. The clinic records of 415 patients examined at two university optometry clinics were examined. The records were selected using systematic random sampling and based on a standardised protocol. NPC was measured using the push-up-to-break technique. The techniques used to measure heterophoria and fusional vergences were not indicated. The authors reported that $33 \%$ of the participants had low suspect CI, $12 \%$ had high suspect and $6 \%$ had definite CI. About $17.6 \%$ had CI with 2 or more signs (classified as clinically significant CI). A major limitation inherent in data derived from an optometry training school where data are collected by different student clinicians is the possibility of inter-examiner variations that could minimise the validity of the study. In another clinic-based study, White and Major ${ }^{42}$ retrospectively analysed 129 cases of patients seen at the vision therapy clinic at the Northeastern State University College of Optometry in Oklahoma. The reported prevalence of CI in children aged $7-19$ years was $7.75 \%$ for clinically significant CI.
Data were collected from independent optometric practices in some studies. In 1992, Dwyer ${ }^{26}$ studied 'vergenceaccommodative' disorders in 144 consecutive patients, aged 7-18 years, who were examined in his optometry practice. CI was classified as uncompensated exophoria and the prevalence estimate was 33\%. Using the same criteria for exophoria at near (low suspect CI), the reported frequency $(33 \%)$ was the same as the findings reported by Rouse et al. ${ }^{38}$ for low suspect CI where data were derived from two university optometry clinics. The high prevalence from both studies ${ }^{25,26}$ relates to the clinical settings from which the data were obtained, as people with visual symptoms are more likely to consult for an eye examination than patients without symptoms. A possible limitation of the study by Dwyer ${ }^{26}$ is that, although several diagnostic signs were outlined, restricted criteria of an uncompensated heterophoria were applied to diagnose CI. Dusek et al. ${ }^{18}$ reported that $5.2 \%$ of 328 patients aged 6-14 years who attended an optometric practice in Austria had CI. Convergence was assessed using the push-up-to-break technique with a penlight target.

Taken together, for CI types 2 and 3 (classified as clinically significant $\mathrm{CI}$ ), the $18 \%$ prevalence reported by Rouse et al. $^{38}$ is similar to the $17.3 \%$ prevalence reported by Borsting et $a .^{41}$ in a non-clinical sample of schoolchildren but higher than the findings from other studies (Rouse et al. [8.4\%], ${ }^{37}$ Rouse et al. [13\%],,$^{39}$ Marran et al. [14.7\%], ${ }^{44}$ White and Major $\left.[7.75 \%]^{42}\right)$. The fact that data from Rouse et al. ${ }^{38}$ was derived from a clinical setting may account for the relatively higher prevalence reported, but the reason for the similarities between the findings of $33 \%$ for low suspect CI reported by Rouse et al. ${ }^{37}$ and Rouse et al. ${ }^{38}$ is unclear. Using single criteria, Dwyer ${ }^{26}$ found a $33 \%$ prevalence of CI in an Australian clinical population. Consequently, it appears that studies conducted on clinical samples had higher prevalence estimates than non-clinic-based studies. The findings from studies on convergence insufficiency are summarised in Table 2. 
TABLE 3: Convergence excess.

\begin{tabular}{llccc}
\hline Authors & $\begin{array}{l}\text { Country of } \\
\text { study }\end{array}$ & Age (years) & $\begin{array}{c}\text { Sample } \\
\text { size }\end{array}$ & $\begin{array}{c}\text { Prevalence } \\
(\mathbf{\%})\end{array}$ \\
\hline Borsting et al. (2003) & America & $8-15$ & 392 & 0.8 \\
Marran et al. (2006) & USA & 11.5 & 299 & 5 \\
Shin et al. (2009) & South Korea & $9-13$ & 114 & 2.4 \\
Scheiman et al. (1996) $)^{25}$ & USA & $6-18$ & 1650 & 7.1 \\
Dwyer (1992) & Australia & $7-18$ & 144 & 15 \\
Dusek et al. (2010) & Austria & $6-14$ & 328 & 8.2 \\
$\begin{array}{l}\text { Wajuihian and Hansraj } \\
(2014)^{28}\end{array}$ & South Africa & $13-19$ & 65 & 3.2 \\
\hline
\end{tabular}

\section{Convergence excess and fusional vergence dysfunction}

CEs and FVD are less prevalent vergence anomalies than $\mathrm{CI}^{12,13}$ and have essentially been reported by the same studies on $\mathrm{CI} .^{12,13} \mathrm{CE}$ is a binocular vision anomaly characterised by a tendency for an individual to over-converge their eyes at near. ${ }^{13,62}$ The clinical diagnostic signs include esophoria greater at near than at distance, reduced negative fusional vergence at near, and high $\mathrm{AC} / \mathrm{A}$ ratio using the gradient method. ${ }^{13,41}$ Other clinical signs include moderate hyperopia, low positive relative accommodation, difficulty with minus lenses on binocular accommodative facility, and high findings on the monocular estimation method of near retinosocopy. ${ }^{13,55}$ The lowest prevalence $(0.8 \%)$ of $\mathrm{CE}$ was reported by Borsting et al. ${ }^{58}$ in a sample of 392 schoolchildren aged between 8 and 15 years selected from four elementary schools. Shin et al. ${ }^{55}$ found that $2.4 \%$ of a sample of 114 South Korean schoolchildren aged between 9 and 13 years had CE whilst Marran et al. ${ }^{44}$ reported a higher $(5 \%)$ prevalence in a sample of 299 schoolchildren (mean age, $11.5 \pm 0.63$ years). In Wajuihian and Hansraj, ${ }^{28}$ the prevalence of CE was 3.2\% and no participants had FVD.

A relatively higher prevalence of CE was reported in four studies. In a clinical sample of schoolchildren, Scheiman et al. ${ }^{25}$ reported a prevalence of $7.1 \%$ which was significantly higher with age and in black children than in white. In the study by Dusek et al., ${ }^{18} 8.2 \%$ of 328 clinical patients (aged 6-14 years) who attended an optometric practice in Austria had CE. Dwyer ${ }^{25}$ reported a CE prevalence of $15 \%$ in 144 consecutive patients (ages 7-18 years) examined at the author's optometry practice. The findings from studies on convergence excess are summarised in Table 3.

\section{Fusional vergence dysfunction}

FVD describes a binocular anomaly whereby there are deficiencies in the fusional vergence dynamics ${ }^{12,13}$ characterised by inability of the fusional vergence system to respond rapidly and accurately to changing vergence demands over time. ${ }^{12,13,35}$ A literature search revealed that FVD has not been studied extensively. Scheiman et al. ${ }^{25}$ reported a $0.4 \%$ prevalence of FVD in children aged between 6 and 18 years. Somewhat related to FVD, a study in South Africa by Metsing and Ferreira ${ }^{59}$ found that $17 \%$ of the participants had poor convergence amplitude and $21.9 \%$ had poor vergence facility.

\section{Convergence insufficiency, gender, and age}

CI does not seem to have any gender preponderance in any age group, as available studies found no significant difference in frequency of CI between male and female subjects. ${ }^{25,39,41}$ The study by Letourneau and Ducic ${ }^{57}$ found CI to be marginally more frequent in girls $(2.1 \%)$ than boys $(1.9 \%)$ in a population of Canadian schoolchildren. The prevalence of CI increased with age in some studies ${ }^{25,43,54}$ whereas Letourneau et al. ${ }^{56}$ found no correlation between CI and age. Similarly, age appears to influence the prevalence estimates of $\mathrm{CE}$ as studies with a higher age range reported a relatively higher prevalence. ${ }^{18,25,26}$

\section{Convergence insufficiency and accommodation insufficiency}

Pseudoconvergence insufficiency (PCI) has been described as a vergence anomaly where the patient has accommodative insufficiency associated with CI. ${ }^{39}$ In normal eyes, accommodation and convergence are neurologically coupled. ${ }^{31,44}$ Convergence results from, and is associated with, any accommodation effort; hence contraction of the ciliary muscles to mediate accommodation is accompanied

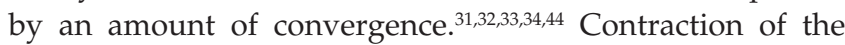
ciliary muscles may be insufficient or excessive but convergence must be exact for any point closer than infinity to avoid diplopia. ${ }^{31}$ Owing to this stable and positive link between accommodation and convergence, ${ }^{33,34}$ a deficiency in one variable may affect the other. ${ }^{31,44} \mathrm{PCI}$ has been described in the literature ${ }^{39,44}$ as a vergence anomaly that results from a person's inability to convergence as a result of insufficient accommodative ability..$^{12}$ This syndrome has been attributed to a central nervous defect that affects the accommodative and convergence mechanism. ${ }^{29,30,39,44}$ Reports on the gender distribution of PCI are mixed. PCI has been recognised as a separate and unique clinical condition..$^{29,30,39,44}$ Marran et al. ${ }^{44}$ reported a $3.3 \%$ prevalence of PCI. The syndrome may be a therapeutic challenge as conventional treatment for CI does not completely resolve it, unless some accommodative therapies are initiated..$^{39,44}$

\section{Convergence insufficiency and ethnicity and race}

On the association of CI with race and ethnicity, only the study by Rouse et al. ${ }^{39}$ reported on the frequency of CI in black and white populations, and found that ethnicity appeared to be significantly associated with CI classification in a study of Hispanic, Asian, white and black people. According to the same study, the small number of Hispanic and Asian children in the study might limit interpretation. Black subjects had a higher frequency $(4.9 \%)$ of definite CI than did white (4.2\%).

\section{Heterophoria}

Heterophoria (abbreviated as phoria) is the relative deviation of the visual axes when the eyes are dissociated. ${ }^{63,64}$ This latent 
TABLE 4: Summary of studies on heterophoria at near.

\begin{tabular}{|c|c|c|c|c|}
\hline Authors & Country of study & Age (years) & Sample size & Prevalence $(\%)$ \\
\hline Aring et al. $(2005)^{52}$ & Sweden & $4-15$ & 143 & Exo 22; Eso 3.5 \\
\hline Leone et al. $(2010)^{48}$ & Australia & $6-9$ & 4093 & Exo 52.2-58.3; Eso 9.2-10.4 \\
\hline Dusek et al. $(2010)^{18}$ & Austria & $6-14$ & 328 & 0.6 horizontal; phoria only \\
\hline Abdi et al. $(2008)^{54}$ & Sweden & $6-16$ & 216 & Exo $8.8 \%$; Eso $1.4 \%$ \\
\hline Walline et al. $(1998)^{46}$ & USA & $\mathrm{n} / \mathrm{a}$ & 1495 & Exo $21 \%$; Eso $12.25 \%$ \\
\hline Junghans et al. $(2002)^{51}$ & Australia & $3-12$ & 2697 & Exo $\geq 9=2.6 \% ;$ Eso $>3=11.9 \%$ \\
\hline Walters $(1984)^{49}$ & Australia & $n / a$ & 5597 & Exo $>5=7.99 \%$; Eso $\geq 3=4.58 \%$ \\
\hline Harris $(2002)^{43}$ & USA & $\mathrm{n} / \mathrm{a}$ & 171 & Exo $>10=7.6 \%$; Eso $>10=2.33 \%$ \\
\hline
\end{tabular}

Eso, Esophoria; Exo, Exophoria.

deviation requires fusional vergence efforts to maintain single binocular vision and involves the accommodation mechanism at near viewing distance. ${ }^{19,64}$ During near tasks, the resulting near phoria measures the degree to which the eyes over-converge (esophoria) or under-converge (exophoria) in relation to the plane of the target. ${ }^{65}$ To attain binocular single vision, exophoric conditions necessitate an increase in fusional convergence (resulting in a convergent vergence adaptation) whilst an esophoric deviation requires an increase in fusional divergence (resulting in a divergent vergence adaptation) to attain binocular single vision. ${ }^{66}$ Accordingly, smooth functioning of accommodative, vergence and ocular alignment is important for efficient near tasks. ${ }^{19}$ Invariably, heterophoria indicates that a person is applying fusional vergence efforts to overcome the dissociated deviation, which is a normal characteristic of binocular status for most people. However, in some cases, the fusional vergence effort could be excessive and could result in symptoms or suppression in which it is described as being decompensated or uncompensated. ${ }^{64}$ In decompensated heterophoria, the vergence eye movement is unable to overcome a heterophoria adequately. ${ }^{64}$

\section{Previous studies on heterophoria Exophoria}

Various studies ${ }^{18,46,48,49,51,52,53,54}$ have reported on heterophoria; four measured phoria using the CT; three $18,48,52$ of the four studies were similar in the use of CT and the classification criteria of two pd for horizontal phoria; one study ${ }^{51}$ used Howell phoria cards and another ${ }^{49}$ measured phoria using the modified Prentice technique. The focus of the present review is on horizontal heterophoria (exophoria and esophoria). The studies that measured phoria using the CT and classified phoria as $2 \mathrm{pd}$ include a prospective cross-sectional study by Aring et al. ${ }^{52}$ on 143 Swedish children of ages 4-15 years in 2005. Aring et al. ${ }^{52}$ reported that $22 \%$ of the participants had exophoria. Neither age nor gender distribution of heterophoria was reported. Leone et al. $^{48}$ conducted a study to determine the prevalence of heterophoria and its associations with refractive errors and ethnicity in 4093 Australian schoolchildren between 6 and 9 years old, and found that $58.3 \%$ of the 6 -year-olds had near exophoria whilst $52.2 \%$ of the 12-year-olds manifested near exophoria. Myopia was found to be associated with exophoria at near, and the average magnitude of prevalent heterophoria at near fixation was reported to be lower in the 12-year-old (3.9 pd) than in the 6-year-old children $(4.9 \mathrm{pd})$. There were no significant gender differences in heterophoria, apart from 6-year-old girls having more exophoria at near fixation although the difference was not significant. A study on Austrian schoolchildren by Dusek et al. $^{18}$ assessed heterophoria using the CT. Only $0.6 \%$ of the participants had horizontal heterophoria. The authors failed to specify whether the horizontal heterophoria was exophoria or esophoria.

The criteria applied to define heterophoria in the following studies were mixed, which makes comparison difficult. Junghans et al. ${ }^{51}$ studied functional vision anomalies in 2687 primary schoolchildren in Australia. Heterophoria was evaluated at $33 \mathrm{~cm}$ for near using the Howell phoria cards technique. Junghans et $a 1 .{ }^{51}$ found that at near, $1.3 \%$ of children had exophoria $>9 \mathrm{pd}$. Five $(0.55 \%)$ of the 896 children tested had vertical phoria at near. In 1984 , Walters ${ }^{49}$ performed a vision screening on 5597 Australian schoolchildren. Heterophoria was assessed at near $(33 \mathrm{~cm})$ using the modified Prentice test, and it was reported that at near, 5.68\% had exophoria $\geq 5 \mathrm{pd}$ and $2.31 \%$ had exophoria $\geq 8$ pd. Neither age nor gender distribution of heterophoria was reported. Harris ${ }^{43}$ studied vision anomalies in a randomly selected sample of elementary schoolchildren in Baltimore City. Heterophoria was assessed using the CT. For near heterophoria, $7.6 \%$ of the children had exophoria $>10 \mathrm{pd}$. Although a less stringent criterion was applied, a higher prevalence was reported in the study by Abdi et al. ${ }^{54}$ on Swedish schoolchildren where the CT was used to assess the angle of heterophoria and exophoria was defined as $\geq 6 \mathrm{pd}$ at near. The prevalence of near exophoria was $8.8 \%$. Neither age nor gender distribution of heterophoria was reported. The highest prevalence was reported by Walline et al. ${ }^{46}$ who studied development of heterophoria in 1495 American schoolchildren. The prevalence of exophoria ranged between $21 \%$ and $31.8 \%$ amongst kindergarten and fifth graders. It is unclear what criteria Walline et al. ${ }^{46}$ used to define heterophoria, although a decrease in exophoria with age was also reported. Several reviewed studies $18,43,48,49,51,54$ found no significant change in phoria at near with increasing age. The prevalence estimates for studies on heterophoria are summarised in Table 4.

\section{Esophoria}

The reports on esophoria are from the same studies that reviewed exophoria. ${ }^{18,46,48,49,51,52,53,54}$ Using criteria of $\geq 2$ pd for Swedish children between 4 and 15 years old, Aring et al. ${ }^{52}$ 
reported a 3.5\% prevalence of esophoria. In the study by Leone et al., ${ }^{48}$ the prevalence of esophoria was $9.2 \%$, and was $10.4 \%$ for children of age 6-12 years respectively. Also, hyperopia was found to be associated with esophoria at near. Abdi et al ${ }^{54}$ defined esophoria as $\geq 4 \mathrm{pd}$ at near and found a $1.4 \%$ prevalence of near esophoria. A higher prevalence of heterophoria was reported by Walline et al., ${ }^{46}$ who found that the prevalence of esophoria increased with age from $6.7 \%$ for kindergarten children and $12.25 \%$ for fifth graders. In the study by Junghans et al. ${ }^{51} 11.9 \%$ of the children had $>3 \mathrm{pd}$ esophoria whilst $0.5 \%$ had $>5$ pd esophoria at near. In another study in Australia, Walters ${ }^{49}$ reported that $4.58 \%$ of the children had esophoria $\geq 3 \mathrm{pd}$, whilst a study by Harris ${ }^{43}$ found that $2.33 \%$ of the participants in a study of American schoolchildren had esophoria.

\section{Discussion}

In the present paper, the prevalence of vergence anomalies was reviewed. The findings from the reviewed studies are summarised in Tables 2-5. Although the reviewed studies provide workable prevalence estimates of vergence anomalies, the vast variations in the clinical measurement techniques and findings from the reviewed studies make it difficult to interpret and apply the findings consistently. In the following paragraphs, the possible sources of variations and limitations of previous studies are outlined. It is hoped that identifying the problems will serve as a guide towards improving future studies.

\section{Sources of variation}

The methodological sources of variations identified from previous studies include:

- differences in instrumentation and techniques

- differences in classification criteria and the number of diagnostic signs

- differences in data analysis methods

- differences in the type of population studied (clinical/ non-clinical)

- representativeness of the populations (probability/nonprobability sampling methods)

- inter-examiner variations

- participants' factors that include age as well as the fact that some techniques such as fusional vergence measurements are mainly subjective

- changes in the nature of near point tasks may be a source of variation in findings across studies.

\section{Limitations of previous studies}

- Most studies failed to detail their sampling strategies and how sample sizes were derived whilst some studies used small sample sizes with a consequently low statistical power. Only two studies ${ }^{38,57}$ indicated how sample sizes were derived. Specifically, sample size determination is important to ensure that the study has a good chance of detecting a statistically significant result if the effect is true that minimises a type two error (chances of missing a significant difference), ${ }^{67,68,69}$ thereby enhancing the generalisability of study findings.

- The eligibility criteria (inclusion and exclusion) that are important in understanding the sample studied, and interpreting and assessing the study validity, ${ }^{23,45}$ were not indicated in some studies.

- In some studies, ${ }^{18,25,26,38}$ samples were derived from clinical or conveniently selected samples. Although some study settings can only permit retrospective and convenience sampling methods which are relevant in providing baseline information, a drawback with such studies is that the reported prevalence may be biased because the study design does not provide for control of bias. In most cases, conveniently selected samples would not permit probability sampling, and most statistical formulas are not designed for samples selected conveniently.

- Another limitation is that some studies failed to either indicate the diagnostic criteria or specify the clinical signs used to define the outcome variable. Failure to report these parameters might affect the internal validity of a study and limit the interpretations of study findings. Moreover, it is important to note that it is rare to find a study without inherent limitations. ${ }^{67,68,69}$ Most reviewed studies failed to indicate the limitations of the studies. Indicating the limitations of a study will enable improvements to subsequent studies.

- Invariably, some conditions such as CI have been studied extensively whereas studies on other anomalies such as convergence excess, fusional vergence dysfunction and heterophoria were less studied.

- Overall, there are limited data regarding how ethnicity, race, age, gender, geographic location, and socioeconomic status influence vergence anomalies. Similarly, there are limited studies from black populations.

- No study could be found that reported on the syndromes of vergence anomalies in any black South African population. Remarkably, only two studies ${ }^{25,26}$ had participants of up to 18 years' age (high school age). The high school populations are particularly relevant for studying as they are vulnerable to accommodative and vergence anomalies because of the relatively high near task demands they are exposed to, given that near tasks increase with increasing school grade level and as children prepare for tertiary education, which has even greater near task demands.

\section{Significance and limitations of present review}

A major strength of the present paper is an extensive and up-to-date review of available literature on vergence anomalies in school-age children. A possible limitation is that only studies reported in the English language were retrieved; it is possible that articles reported in other languages were omitted. Secondly, only published papers were reviewed; credible unpublished studies might have been omitted. Despite the outlined limitations, the present review is significant and has important implications and applications in optometric education, clinical practice, research and health policy planning. 


\section{Recommendations for future studies}

The following recommendations are aimed to improve future research on accommodative and vergence anomalies:

- To minimise variations in classifications and investigative techniques, international expert bodies such as the convergence insufficiency and reading study group and the Convergence Insufficiency Treatment Trial groups should stipulate a standardised study design (common definitions, measuring techniques and diagnostics criteria, statistical analyses and reporting methods) for accommodative and vergence anomalies. Such criteria should be used as a benchmark to access credibility of subsequent studies. Consistent diagnostic techniques and criteria are important, considering their impact on diagnosis and treatment.

- Vergence anomalies are prevalent in school-age populations and, given the clinical significance, it is important that optometrists and other eye care professionals consider it mandatory to evaluate, diagnose and treat these anomalies in all settings. Continued research by professionals should also be considered as a major aspect of interest.

- Further research is needed as to whether the prevalence of accommodative and convergence anomalies varies by ethnicity, race, age, sex, geographic location or socioeconomic status. These factors can be investigated via collaborations between international researchers.

- Studies on high school populations will be relevant to characterise vergence anomalies in such populations.

- Authors should adequately detail all testing protocols to enable reproducibility of a study by other researchers.

- Studies with larger sample sizes using random sampling would yield more conclusive results.

\section{Summary and conclusion}

Summaries of the reviewed studies are presented in Tables 2-5. Irrespective of whether single or multiple clinical signs were used to classify vergence anomalies, the present review shows that these anomalies are prevalent in all settings in school-age populations. The lack of uniform diagnostic protocol and classification criteria has complicated the comparison of prevalence estimates from different studies. Consequently, the present review has provided a workable prevalence range for vergence anomalies and highlighted some methodological concerns in previous studies. Given that treatment regimens are based on appropriate measurement and diagnosis, it is important to establish a standardised diagnostic protocol and criteria with the ultimate goal of optimum patient care. Continued discourse, which will further enhance our understanding of vergence anomalies, is certainly needed, given that the negative consequences of untreated vergence anomalies are numerous. Optometry plays a major role in the detection, assessment and management of visual anomalies in children, as the optometrist is often one of the healthcare professionals whom parents consult when a child performs poorly academically.
TABLE 5: Summary range of prevalences for vergence anomalies.

\begin{tabular}{lc}
\hline Range of prevalences for vergence anomalies & Prevalences (\%) \\
\hline $\begin{array}{l}\text { Convergence insufficiency based on single definition } \\
\text { of receded NPC }\end{array}$ & $6-18$ \\
$\begin{array}{l}\text { Convergence insufficiency in non-clinical populations } \\
\text { of schoolchildren }\end{array}$ & $6-28$ \\
$\begin{array}{l}\text { Convergence insufficiency in clinical populations of } \\
\text { schoolchildren }\end{array}$ & $0.8-33$ \\
Convergence insufficiency based on multiple criteria & $2.1-33$ \\
$\begin{array}{l}\text { Clinically significant convergence insufficiency } \\
\text { (Cl } 2 \text { and } \mathrm{Cl} 3 \text { ) based on multiple criteria }\end{array}$ & $0.8-28$ \\
Convergence excess in school setting & $0.8-5$ \\
Convergence excess in clinic setting & $7.1-20.83$ \\
Fusional vergence dysfunction & $0-0.4$ \\
Exophoria ( $\geq 2$ pd) & $0.6-58.3$ \\
Exophoria ( $\geq 5$ pd) & $2.6-21$ \\
Esophoria ( $\geq 2$ pd) & $2.3-10.4$ \\
Esophoria ( $\geq 3$ pd) & $1.4-11.9$
\end{tabular}

NPC, near point of convergence.

\section{Acknowledgement}

The authors are grateful to Professor B.M. Zungu and the external referees for reviewing the manuscript.

\section{Competing interests}

The authors declare that they have no financial or personal relationships which may have inappropriately influenced them in writing this article.

\section{Authors' contributions}

S.O.W. (University of KwaZulu-Natal) wrote the manuscript comprising this review. R.H. (University of KwaZulu-Natal) contributed intellectually overall and played a supervisory role.

\section{References}

1. Garzia R. The relationship between visual efficiency problems and learning In: Scheiman $M$, Rouse $M$, editors. Optometric management of learning-related vision problems. 1st ed. St. Louis, MO: Mosby, 2006; pp. 209-280.

2. Hoffman LG, Rouse M. Referral recommendations for binocular function and/or developmental perceptual deficiencies. J Am Optom Assoc. 1980;51: 119-125.

3. Bodack M, Chun I, Krumholtz I. An analysis of vision screening data from New York City public schools. Optometry. 2010;81:476-484. http://dx.doi.org/10.1016/j. optm.2010.05.006

4. Cacho-Martínez P, García-Muñoz A, Ruiz-Cantero MT. Do we really know the prevalence of accommodative and nonstrabismic binocular dysfunctions? J Optom. 2010;3:185-197. http://dx.doi.org/10.1016/S1888-4296(10)70028-5

5. Birnbaum M. Symposium on near point visual stress. Introduction. Am J Optom Arch Am Acad Optom. 1985;62:361-364. http://dx.doi.org/10.1097/00006324198506000-00001

6. Vellutino FR, Fletcher JM, Snowling MJ, Scanlon DM. Specific reading disability (dyslexia): What have we learned in the past four decades? J Child Psycho Psychiatry. 2004;45:2-40. http://dx.doi.org/10.1046/j.0021-9630.2003.00305.x

7. Ritty JM, Solan HA, Cool SJ. Visual and sensory-motor functioning in the classroom: A preliminary report of ergonomic demands. J Am Optom Ass. 1993;64: 238-244.

8. Gallaway M, Mitchell LG. Validity of the VERA visual skills screening. Optometry. 2010;81:571-579. http://dx.doi.org/10.1016/j.optm.2010.07.024

9. GranetD, GomiC, Ventura R, Miller-ScholteA. The relationship between convergence insufficiency and ADHD. Strabismus. 2005;13:163-168. http://dx.doi.org/10.1080/ 09273970500455436

10. Borsting $E$, Rouse $M$, Chu R. Measuring ADHD behaviours in children with symptomatic accommodative dysfunction or convergence insufficiency: A preliminary study. Optometry. 2005;76:588-592. http://dx.doi.org/10.1016/j. optm.2005.07.007

11. Scheiman M. Rouse M. Optometric management of learning-related vision problems. 2nd ed. St. Louis, MO: Mosby, 2006. 
12. American Optometric Association. Optometric clinical practice guidelines: care of the patient with accommodative and vergence dysfunctions [serial on the Internet]. 2013 [cited 15 January 2013]. Available from: http://www.aoa.org/ documents/CPG-20

13. Scheiman M, Wick B. Clinical management of binocular vision: Heterophoric, accommodative and eye movement disorders. 3rd ed. Philadelphia, PA: JB Lippincott, 2008

14. Zaba JN. Social, emotional and educational consequences of undetected children's vision problems. J Behav Optom. 2001;12:66-70.

15. Gronlund MA, Aring E, Landren M, Hellstro A. Visual function and ocular features in children and adolescents with attention deficit hyperactivity disorder, with and without treatment with stimulants. Eye. 2007;21:494-502.

16. Damari DA, Liu J, Smith KB. Visual disorders misdiagnosed as ADHD. Case studies and literature. J Behav Optom. 2000;11:87-91.

17. Johnson RA, Nottingham $D$, Stratton R, Zaba J. The vision screening of academically and behaviorally at-risk pupils. J Behav Optom. 1996;71:39-42.

18. Dusek W, Piersionek BK, McClelland JF. A survey of visual functions in an Austrian population of school-age children with reading and writing difficulties. BMC Ophthalmol. 2010;10:1-10. http://dx.doi.org/10.1186/1471-2415-10-16

19. Simons HD, Grisham JD. Binocular anomalies and reading problems. J Am Optom Assoc. 1987; 58:578-586.

20. Palomo-Álvarez C, Puell MC. Accommodative functions in schoolchildren with reading difficulties. Graefe's Arch Clin Exp Ophthalmol. 2008;246:1769-1774. http://dx.doi.org/10.1007/s00417-008-0921-5

21. Palomo-Alvarez C. Binocular functions in school children with reading difficulties. Graefe Arch Clin Exp Ophthalmol. 2010;248:885-892. http://dx.doi.org/10.1007/ s00417-009-1251-y

22. Wajuihian SO, Naidoo KS. A review of visual factors S Afr Optom. 2010;69: 58-68.

23. Hulley SB, Cummings SR, Browner WS, Grady DG, Newman. TB. Designing clinical research. 3rd ed. Philadelpia, PA: Lippincott, 2007.

24. Bennett GR, Blondin M, Ruskiewics J. Incidence and prevalence of selected visual conditions. J Am Optom Assoc. 1982;53:647-656.

25. Scheiman M, Gallaway M, Coulter R, Reinstein F, Ciner E. Prevalence of vision and ocular disease conditions in a clinical pediatric population. J Am Optom Assoc. 1996;67:193-202.

26. Dwyer $P$, Wick B. The influence correction upon disorders of vergence and accommodation. Optom Vis Sci. 1995;72:224-232. http://dx.doi.org/10.1097/ 00006324-199504000-00002

27. Cooper J, Jamal N. Convergence insufficiency - a major review. Optometry. 2012;83:137-158.

28. Wajuihian SO, Hansraj R. Near vision anomalies in a population of Black high schoolchildren in South Africa: A pilot study. S Afr Optom. 2014;73:21-32.

29. Duane A. Convergence insufficiency. J Am Med Assoc. 1914;LXIII:721-729. http:// dx.doi.org/10.1001/jama.1914.02570090007003

30. Mann I. Convergence deficiency. Br J Ophthalmol. 1940;24:373-390. http:// dx.doi.org/10.1136/bjo.24.8.373

31. Dobson M. Convergence. $\mathrm{Br} J$ Ophthalmol. 1941;25:66-71. http://dx.doi. org/10.1136/bjo.25.2.66

32. Morgan MW. The clinical aspects of accommodation and convergence. Am J Optom Arch Am Ac Optom. 1944;21:301-313. http://dx.doi.org/10.1097/00006324 194408000-00001

33. Morgan MW. Accommodation and vergence. Am J Optom Arch Am Acad Optom. 1968;45:417-454. http://dx.doi.org/10.1097/00006324-196807000-00002

34. Fink W. Symposium: convergence insufficiency. Am Orth J. 1953;3:5-26.

35. Eskridge JB, Amos JF, Bartlett JD. Clinical procedures in optometry. New York: JP Lippincott, 1991

36. Lavrich JB. Convergence insufficiency and its current treatment. Current Opin Ophthalmol. 2010;21:356-360. http://dx.doi.org/10.1097/ICU.0b013e32833cf03a

37. Rouse MW, Borsting E, Hyman L, Hussein M. Pilot study to evaluate convergence insufficiency in a school-aged population: Poster \# 54 (BV-323). Optom Vis Sci. 1995;72:218. http://dx.doi.org/10.1097/00006324199512001-00353

38. Rouse M, Hyman L, Hussein M, Solan H. Frequency of convergence insufficiency in optometry clinic settings. Convergence insufficiency and reading study (CIRS) Group. Optom Vis Sci. 1998;75:88-96. http://dx.doi.org/10.1097/00006324199802000-00012

39. Rouse MW, Borsting E, Hyman L, Hussein M, Solan H. Frequency of convergence insufficiency in optometry clinic settings. Optom Vis Sci. 1999;76:643-649. http:// dx.doi.org/10.1097/00006324-199909000-00022

40. Borsting EJ, Rouse MW, Mitchell GL, Scheiman M, Cotter SA, Cooper J. Validity and reliability of the revised convergence insufficiency symptom survey in children aged 9 to 18 years. Optom Vis Sci. 2003;80:832-838. http://dx.doi. org/10.1097/00006324-200312000-00014
41. Borsting E, Rouse MW, Deland PN, Hovett S, Kimura D, Park M, et al. Association of symptoms and convergence and accommodative insufficiency in school-age of symptoms and convergence and
children. Optometry. 2003;74:25-34.

42. White T, Major A. A comparison of subjects with convergence insufficiency and subjects with normal binocular vision. J Behav Optom. 2004;15:37-41.

43. Harris P. Learning-related visuals problems in Baltimore city: A long-term program J Optom Vis Dev. 2002;33:75-115.

44. Marran LF, De Land PN, Nguyen AL. Accommodative insufficiency is the primary source of symptoms in children diagnosed with convergence insufficiency. Optom Vis Sci. 2006;83:E281-E289. http://dx.doi.org/10.1097/01. opx.0000216097.78951.7b

45. Daum KM. Characteristics of convergence insufficiency. Am J Optom Physiol Opt. 1988;65:426-438. http://dx.doi.org/10.1097/00006324-198806000-00002

46. Walline JJ, Mutti DO, Zadnik K. Development of phoria in children. Optom Vis Sci. 1998;75:605-610. http://dx.doi.org/10.1097/00006324-199808000-00026

47. Amster D. Fusional vergence dysfunction. J Behav Optom. 2008;19:59-62.

48. Leone JF, Cornell E, Morgan IG, Mitchell P, Kifley A, Wang JJ, et al. Prevalence of heterophoria and associations with refractive error, heterotropia and ethnicity in Australian school children. Br J Ophthalmol. 2010;94:542-546. http://dx.doi. org/10.1136/bjo.2009.163709

49. Walters J. Portsea modified clinical technique: results from an expanded optometric screening protocol for children. Aust J Optom. 1984;67:176-218. http://dx.doi.org/10.1111/j.1444-0938.1984.tb03729.x

50. Macfarlane DJ, Fitzgerald WJ, Stark DJ. The prevalence of ocular disorders in 1000 Queensland primary schoolchildren. Aust N Z J Ophthalmol. 1987;15:161-174. http://dx.doi.org/10.1111/j.1442-9071.1987.tb00066.x

51. Junghan B, Keily P, Crewther DP, Crewther SG. Referral rates for a functional vision screening among a large cosmopolitan sample of Australian children. Ophtha Physiol Opt. 2002;22:10-25. http://dx.doi.org/10.1046/j.1475-1313.2002.00010.x

52. Aring E, Gronlund MA, Andersson S. Strabismus and binocular functions in a sample of Swedish children aged 4-15 years. Strabismus. 2005;13:55-61. http:// dx.doi.org/10.1080/09273970590922664

53. Abdi S, Rydberg A. Asthenopia in schoolchildren, orthoptic and ophthalmological findings and treatment. Doc Ophthalmol. 2005;111:65-72. http://dx.doi. org/10.1007/s10633-005-4722-4

54. Abdi S, Lennerstrand G, Pansell T, Rydberg A. Orthoptic findings and asthenopia in a population of Swedish schoolchildren aged 6 to 16 years. Strabismus. 2008;16:47-55. http://dx.doi.org/10.1080/09273970802020243

55. Shin HS, Park SC, Park CM. Relationship between accommodative and vergence dysfunction and academic achievement for primary school children. Ophthal Physio Opt. 2009;29:615-624. http://dx.doi.org/10.1111/j.1475-1313.2009.00684.x

56. Letourneau J, Lapierre $N$, Lamont $A$. The relationship between convergence insufficiency and school achievement. Am J Optom Physiol Opt. 1979;56:18-22. http://dx.doi.org/10.1097/00006324-197901000-00004

57. Letourneau J, Ducic S. Prevalence of convergence insufficiency among elementary school children. Can J Optom. 1988:50:194-197.

58. Borsting $E$, Rouse M, De Land P. The prevalence of convergence excess in schoolaged children. (meeting abstract). Optom Vis Sci. 2003;80:169.

59. Metsing IT, Ferreira JT. Accommodation and vergence status among the 3rd and 4th graders in mainstream school in Gauteng. S Afr Optom. 2012;71:22-31.

60. Goss DA, Becker E. Comparison of near fusional vergence ranges with rotary prisms and with prism bars. Optometry. 2011;82:104-107. http://dx.doi. org/10.1016/j.optm.2010.09.011

61. Ciuffreda M, Ciuffreda KJ, Wang B. Repeatability and variability of near vergence ranges. J Behav Optom. 2006;1:31-46.

62. American Optometric Association. Fact sheet on vision therapy [homepage on the Internet]. c2011 [cited 13 February 2013]. Available from: http://www.aoa.org/ documents/VT-Reimbursement-Packet.pdf

63. Dowley D. Heterophoria. Optom Vis Sci. 1990;67:456-460. http://dx.doi. org/10.1097/00006324-199006000-00010

64. Evans BJ. Optometric prescribing for decompensated heterophoria. Optometry in Practice. 2008;9:63-78.

65. Suchoff IB. Research on the relationship between reading and vision - what does it mean? J Learn Disab. 1981;14:573-576.

66. Sreenivasan V, Elizabeth L, Irving EL, Bobier WR. Effect of heterophoria type and myopia on accommodative and vergence responses during sustained near activity in children. Vision Res. 2012;57:9-17. http://dx.doi.org/10.1016/j. visres.2012.01.011

67. Zadnik K. Critically reviewing the ophthalmic literature. Optom Vis Sci. 1994;71:254-258. http://dx.doi.org/10.1097/00006324-199404000-00005

68. Mitchell ML, Jolley JM. Research design explained. 7th ed. Belmont, CA: Wadsworth Publishers; 2010.

69. Miller J. The Chicago guide to writing about numbers. Chicago, IL: University of ChicagoPress;2004.http://dx.doi.org/10.7208/chicago/9780226526324.001.0001 